\title{
Impactos da mudança estrutural da economia brasileira sobre o seu crescimento
}

\author{
Luciano Nakabashi* \\ Fábio Dória Scatolin ${ }^{* *}$ \\ Marcio José Vargas da Cruz ${ }^{* * *}$
}

RESUMO - Na abordagem estruturalista, o papel da indústria tem um destaque, pois vários fatores apontam que esse é um setor essencial para a dinâmica da economia. Hirschman (1958) aponta que a indústria tem um nível mais elevado de encadeamentos para frente e para trás em relação aos demais setores. Adicionalmente, o autor coloca que as externalidades positivas e os efeitos de transbordamentos seriam mais relevantes naquele setor. Kaldor (1957) ainda chama a atenção para o maior potencial dos ganhos de escala estáticos e dinâmicos da indústria. Considerando esses argumentos, o presente estudo busca analisar os efeitos da mudança estrutural da economia brasileira sobre o seu desempenho no período 1948-2007.

Palavras-chave: Crescimento econômico. Mudança estrutural. Modelos auto-regressão vetorial.

\section{INTRODUÇÃO}

Muitos são os fatores apontados na teoria econômica como sendo relevantes na determinação do crescimento econômico de uma nação. Um dos possíveis fatores é a estrutura produtiva de uma determinada economia, sendo este o foco do presente artigo.

$\mathrm{Na}$ abordagem estruturalista, o papel da indústria tem um destaque, pois vários fatores apontam que esse é um setor essencial para a dinâmica da economia. Hirschman (1958) aponta que a indústria tem um nível mais elevado de encadeamentos para frente e para trás em relação à agropecuária e ao setor de serviços. Adicionalmente, o autor coloca que as externalidades positivas e os efeitos de transbordamentos seriam mais relevantes naquele setor. Assim, o seu crescimento teria maiores impactos positivos na economia como um todo. Kaldor (1957) também chama a atenção para o papel diferenciado indústria com relação à tecnologia, além do maior potencial dos ganhos de escala estáticos e dinâmicos.

\footnotetext{
* Doutor em Economia pelo CEDEPLAR/UFMG. Professor do Departamento de Economia da Universidade Federal do Paraná (UFPR), Coordenador Geral do Boletim de Economia e Tecnologia. Endereço eletrônico: luciano.nakabashi@ufpr.br.

** Doutor em Economia pela University of London. Professor do Departamento de Economia da Universidade Federal do Paraná (UFPR). Endereço eletrônico: scatolin@ufpr.br.

*** Mestre em Economia pela Universidade Federal do Paraná. Professor do Departamento de Economia da Universidade Federal do Paraná (UFPR). Endereço eletrônico: marciocruz@ufpr.br.
} 
Considerando esses argumentos, o presente estudo busca analisar a importância da indústria e dos demais setores sobre o crescimento da economia brasileira no período 19482007. Nesse período, o país passou por um processo de mudança estrutural, onde a indústria ganhou participação e importância na dinâmica da economia até meados de 1980. A partir de então, a indústria sofreu uma perda considerável de participação no PIB para iniciar uma modesta recuperação a partir dos anos 2000. A principal questão é: qual a relevância dessa mudança estrutural sobre o desempenho da economia brasileira?

Considerando a literatura internacional sobre o tema, um estudo mais aprofundado para o Brasil, inclusive com a utilização de métodos formais se mostra de grande relevância, ainda mais quando se considera a escassez de estudos para mensurar os impactos da mudança estrutural sobre o dinamismo da economia brasileira, sobretudo da participação da indústria no PIB.

A metodologia para atingir o objetivo proposto é a utilização de modelos de Auto Regressão Vetorial (Vector Auto Regression - VAR), pois ela nos permite verificar os efeitos defasados das variáveis relevantes sem a necessidade de se determinar quais são as variáveis endógenas e exógenas no modelo a priori. Essa é uma grande vantagem devido à incerteza da relação de causalidade em um modelo de mudança estrutural e de investimentos devido à complexidade da relação entre as variáveis consideradas.

Além dessa introdução, o presente artigo apresenta uma segunda seção que trata da metodologia e dos dados utilizados para análise. Por fim, na terceira seção, os resultados empíricos são analisados.

\section{METODOLOGIA E FONTE DE DADOS}

A metodologia dos modelos de Auto Regressão Vetorial (Vector Auto Regression VAR) foi empregada para se analisar a relação de causalidade entre as variáveis consideradas relevantes no presente artigo.

Tal metodologia é adequada quando não se tem certeza de quais são as variáveis endógenas e exógenas do modelo. Adicionalmente, todas podem ser consideradas endógenas, em um primeiro momento, para se analisar a relação de causalidade entre elas (ENDERS, 2004).

Uma das pré-condições para a utilização dessa metodologia é a condição de estacionaridade das séries utilizadas no modelo. Para fazer os testes de estacionaridade, foi 
empregado o teste de Dickey-Fuller Aumentado (Augmented Dickey-Fuller - ADF) para raiz unitária.

As estratégias de estimação utilizadas foram duas. A primeira leva em conta a taxa de variação da formação bruta de capital fixo, como uma proxy dos investimentos em capital físico, além das taxas de variação do PIB da agricultura, da indústria e do setor de serviços. Assim, essa seria uma relação mais simples onde a taxa de crescimento da economia depende da taxa de crescimento de cada um dos setores da economia.

Por sua vez, a taxa de crescimento de cada setor depende da taxa de crescimento defasada de todos os setores da economia (efeitos de economias de escala dinâmica e desenvolvimento tecnológico quando se refere à taxa de crescimento defasada do próprio setor e efeitos de encadeamento quando se refere à taxa de crescimento defasada do próprio setor e dos demais). Assim, a inclusão de defasagens da taxa de crescimento dos diferentes setores visa capturar o efeito estrutural da economia sobre o crescimento.

Outra variável relevante, como ressaltado por Solow $(1956,1957)$, seria o investimento. Por isso, utiliza-se a taxa de variação da formação bruta de capital fixo como uma das variáveis desse modelo. O modelo desenvolvido por Solow (1956) aponta para a importância do investimento em capital físico no processo de crescimento econômico por ampliar a capacidade produtiva em momentos futuros, ou seja, focando o lado da oferta.

No entanto, como enfatizado por Blomström, Lipsey e Zejam (1993), o crescimento econômico gera maiores oportunidades de investimento através da elevação de seus respectivos retornos. Assim, os resultados dão suporte à idéia de que as decisões de investimentos por parte dos empresários são endógenas e dependem das expectativas de retorno de seus projetos.

A segunda abordagem adiciona mais variáveis para se ter uma ideia mais completa da inter-relação entre elas, devido à complexidade das inter-relações existentes. Essas variáveis adicionais são as taxas de variação da formação bruta de capitais fixo de máquinas e equipamentos, do PIB da economia como um todo, do PIB da indústria de transformação e do PIB do comércio.

Desse modo, foram utilizadas oito variáveis econômicas no presente estudo, tendo como base suas respectivas taxas reais de variação: 1) da Formação Bruta de Capital Fixo $(\Delta \mathrm{FBKF}) ; 2)$ da Formação Bruta de Capital Fixo das Máquinas e Equipamentos

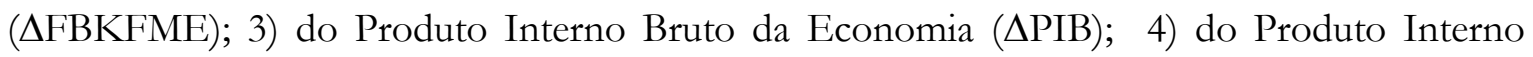
Bruto da Agropecuária $(\Delta \mathrm{PIBA}) ; 5)$ do Produto Interno Bruto da Indústria $(\Delta \mathrm{PIBI}) ; 6)$ do 
Produto Interno Bruto da Indústria de Transformação (APIBIT); 7) Produto Interno Bruto do Setor de Serviços ( $\triangle \mathrm{PIBS}$ ); e 8 ) do Produto Interno Bruto do Comércio ( $\triangle \mathrm{PIBC}$ ).

Todos os dados foram retirados do Sistema de Contas Nacionais do IBGE. A exceção foi a série do PIB da economia em preços constantes de 2007, que tem como fonte o IPEA. Os PIBs da agropecuária, da indústria e do setor de serviços foram calculados a partir do valor adicionado de cada um desses setores como porcentagem do PIB (Sistema de Contas Nacionais do IBGE) multiplicado pelo PIB a preços constantes de 2007 (IPEA).

\section{ANÁLISE ESTATÍSTICA DOS DADOS PARA SÉRIES ANUAIS: 1948-2007}

Nessa seção o foco da análise é o período 1948-2007 para dados anuais. Esse período abrange a fase principal da industrialização da economia brasileira, além da perda de participação da indústria no PIB total a partir de meados dos anos 80 , ou seja, um período em que a economia passou por profundas mudanças estruturais. Desse modo, essa análise nos fornece uma ideia de como essas mudanças afetaram sua própria dinâmica e seu potencial de crescimento.

FIGURA 1 - EVOLUÇÃO DOS PIBS DE DIFERENTES SETORES, SEGMENTOS E DA ECONOMIA COMO UM TODO: $1947-200743$

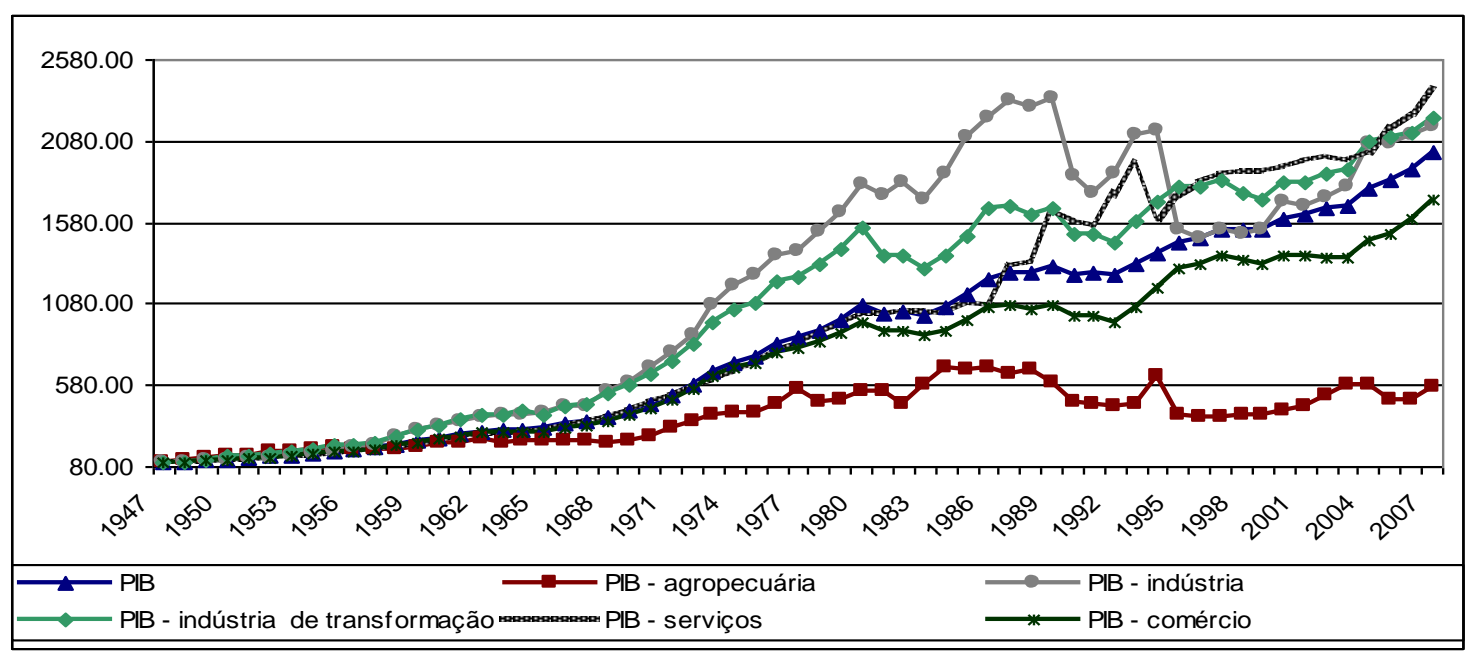

FONTE: elaboração Própria a partir de dados do IBGE/SCN.

NOTAS: os PIBs da economia e da indústria foram normalizados para que os valores em 1948 fossem iguais a $100(1948=100)$.

Na Figura 1 os dados da evolução dos PIBs dos diferentes setores, segmentos e da economia como um todo são apresentados. Pela Figura 1, podemos ver o expressivo avanço dos PIBs da indústria e da indústria de transformação até meados de 80 e uma significativa perda desde então. Essa tendência se reverteu novamente a partir do início dos anos 2000,

43 A série vai de 1947-2007. No entanto, como utilizamos a taxa de crescimento das mesmas na análise econométrica, o período se reduziu para 1948-2007.

92 
quando o PIB de ambos voltou a apresentar taxas de crescimento mais elevadas, inclusive voltando a ganhar participação no PIB total.

A aceleração do crescimento dos PIBs do setor de serviços, a partir de 1985, e do comércio, a partir de 1992, também são fatos importantes. O crescimento do setor de serviços se acelerou justamente quando a indústria perdia dinamismo e, a partir de 1992, o comércio se tornou mais representativo no crescimento. Notamos ainda uma retomada da agropecuária a partir de 1996.

Considerando dados anuais para o período 1948-2007 e seguindo em direção a uma análise mais formal, foram realizados testes de estacionaridade das séries através de testes ADF. A seleção das defasagens e da inclusão ou não de constante e/ou tendência foi feita através do critério de informação de Schwarz (CIS), com a limitação de se utilizar, no máximo, quatro defasagens. Quatro anos parece ser um tempo relativamente longo para se esperar que todos os efeitos relevantes que o crescimento de um setor sobre os diferentes setores e entre os distintos segmentos do mesmo setor terminem. De qualquer forma, a inclusão de oito defasagens não alterou os resultados ${ }^{44}$. Na Tabela 1, podemos visualizar os resultados dos testes:

TABELA 1 - TESTES DE ESTACIONARIDADE DAS SÉRIES - DICKEY FULLER AUMENTADO

\begin{tabular}{l|c|c|c}
\hline \multicolumn{1}{c}{ Variável } & Número de Defasagens & Constante e Tendência & Nível \\
\hline$\Delta$ FBKF & 1 Defasagem & Constante & $-5.56^{* * *}$ \\
$\Delta$ FBKFME & 1 Defasagem & Constante & $-7.32^{* * *}$ \\
$\Delta$ PIB & 1 Defasagem & Constante e Tendência & $-4.78^{* * *}$ \\
$\Delta$ PIBA & 1 Defasagem & Constante & $-7.98^{* * *}$ \\
$\Delta$ PIBI & 1 Defasagem & Constante e Tendência & $-5.84^{* * *}$ \\
$\Delta$ PIBIT & 1 Defasagem & Constante e Tendência & $-6.12^{* * *}$ \\
$\Delta$ PIBS & 1 Defasagem & Constante e Tendência & $-9.12^{* * *}$ \\
$\Delta$ PIBC & 1 Defasagem & Constante & $-4.57^{* * *}$ \\
\hline
\end{tabular}

NOTAS: Hipótese Nula: a variável possui raiz unitária/não é estacionária. A rejeição da hipótese nula é indicada três asteriscos $(1 \%)$. $\triangle \mathrm{FBKF}$ é a taxa de variação da Formação Bruta de Capital Fixo; $\triangle \mathrm{FBKFME} \mathrm{é} \mathrm{a} \mathrm{taxa} \mathrm{de}$ variação da Formação Bruta de Capital Fixo de Máquinas e Equipamentos, $\Delta$ PIB é a taxa de variação do PIB total; $\triangle \mathrm{PIBA}$ é a taxa de variação do PIB da agricultura; $\triangle \mathrm{PIBI}$ é a taxa de variação do PIB da indústria; $\triangle \mathrm{PIBIT}$ é a taxa de variação do PIB da indústria de transformação; $\triangle$ PIBS é a taxa de variação do PIB do setor de serviços; $\triangle$ PIBC é a taxa de variação do PIB comercial. Período 1948 - 2007 para dados anuais.

Como seria de se esperar por estarmos trabalhando com taxas, todas as séries são estacionárias ao nível de 1\%. Desse modo, não haveria problemas em se utilizar os modelos VAR para a análise da relação de causalidade entre as variáveis.

\footnotetext{
44 Os resultados se encontram com os autores. Não apresentamos os resultados com oito defasagens devido ao problema de graus de liberdade no modelo com mais variáveis. Nossa opção foi por utilizar o mesmo (ou quase o mesmo) número de defasagem nas diferentes especificações sem reduzir demasiadamente o número de graus de liberdade em cada uma delas.
} 
$\mathrm{Na}$ Tabela 2, os resultados da estimação do modelo VAR para uma defasagem com as variáveis $\triangle \mathrm{FBKF}, \triangle \mathrm{PIBA}, \triangle \mathrm{PIBI}$ e $\triangle \mathrm{PIBS}$, sendo consideradas como endógenas e com apenas uma defasagem de acordo com a indicação dos critérios de seleção.

Os resultados nos mostram que apenas três coeficientes foram significativos (com exceção das constantes). Dois deles é da primeira defasagem de $\Delta \mathrm{PIBI}$ quando as variáveis dependentes são $\triangle$ PIBA e $\triangle$ PIBI. Isso indica que a taxa de crescimento do PIB industrial defasado em um período tem um impacto estatisticamente significativo sobre a própria taxa de crescimento do PIB industrial, assim como do PIB da agricultura. Além disso, a magnitude do impacto é relevante, pois a elevação de um ponto percentual (p.p.) na taxa de crescimento do PIB industrial do ano anterior tem um impacto de um aumento de 0,46 p.p. em $\triangle$ PIBA e de 0,37 p.p. em $\triangle$ PIBI.

Desse modo, os resultados indicam que existe um maior nível de encadeamento e de mudanças tecnológicas na indústria em relação a outros setores da economia, o que gerou impactos positivos sobre o desempenho da economia no período em questão. Assim, os resultados estão de acordo com Silva e Silveira Neto (2007), que encontraram evidências de importantes encadeamentos para frente e para trás gerados pela indústria e com a análise de Feijó, Carvalho e Rodriguez (2003) que apontaram evidências que mostram a importância da indústria no processo de inovação no aumento de produtividade.

TABELA 2 - RESULTADOS DA ESTIMAÇÃO DO MODELO VAR: 1948 - 2007

\begin{tabular}{lcccc}
\hline & $\Delta$ FBKF & $\Delta$ PIBA & $\Delta$ PIBI & $\Delta$ PIBS \\
\hline$\Delta$ FBKF (L1) & 0.1822 & -0.2391 & -0.1280 & 0.1671 \\
\multirow{2}{*}{$\Delta$ PIBA (L1) } & $(0.141)$ & $(0.168)$ & $(0.121)$ & $(0.087)^{*}$ \\
& 0.0581 & -0.1106 & 0.0308 & 0.0288 \\
$\Delta$ PIBI (L1) & $(0.122)$ & $(0.145)$ & $(0.105)$ & $(0.075)$ \\
& 0.2422 & 0.4615 & 0.3763 & 0.0162 \\
$\Delta$ PIBS (L1) & $(0.192)$ & $(0.229)^{* *}$ & $(0.165)^{* *}$ & $(0.119)$ \\
CONS & 0.0399 & 0.3619 & 0.1968 & -0.1352 \\
& $(0.234)$ & $(0.279)$ & $(0.201)$ & $(0.145)$ \\
& 0.0259 & 0.0030 & 0.0294 & 0.0527 \\
\end{tabular}

NOTAS: desvio-padrão entre parênteses. Um (1) asterisco indica que o coeficiente é significativo ao nível de $10 \%$, dois (2) indicam que é ao nível de $5 \%$, enquanto três (3) indicam significância ao nível de $1 \%$. Variáveis consideradas endógenas: $\triangle \mathrm{FBKF} ; \triangle \mathrm{PIBA} ; \triangle \mathrm{PIBI} ; \triangle \mathrm{PIBS}$. De acordo com os critérios de seleção, foi utilizada apenas uma defasagem. Período 1948 - 2007 para dados anuais. Especificação com menos variáveis. 
O outro coeficiente estatisticamente diferente de zero é o da variável $\triangle \mathrm{FBKF}$, o que indica que o investimento em capital fixo beneficiou, principalmente, o setor de serviços da economia.

Como a relação pode ser mais complexa, introduzimos novas variáveis no modelo inicial para ver a robustez dos resultados, além de verificar uma relação mais complexa que pode trazer insights sobre o complexo processo de mudança estrutural e crescimento econômico.

Por exemplo, o impacto da taxa de variação da Formação Bruta de Capital Fixo de Máquinas e Equipamentos pode ser mais relevante na determinação da taxa de crescimento dos diferentes setores da economia do que a Formação Bruta de Capital Fixo como um todo. Outro seria a possibilidade de uma maior importância de se considerar a indústria de transformação no lugar da indústria como um todo devido à maior dinâmica da primeira, além de um conteúdo tecnológico mais elevado.

O fato de se colocar a taxa de variação do PIB total da economia testa a possibilidade de cada setor afetar o PIB como um todo, além do fato de que este pode afetar cada um dos setores de forma diferenciada. Para completar a análise, introduzimos a taxa de variação do PIB do setor comercial da economia com intuito de verificar se a dinâmica deste se mostra diferente da taxa de variação do PIB do setor de serviços.

Os resultados desse modelo mais complexo se encontram na Tabela 3. Nela, podemos constatar que a primeira defasagem de $\Delta$ PIBI tem impactos positivos e significativos sobre $\triangle$ FBKFME, $\triangle$ PIBIT e $\triangle$ PIBC. Seu impacto também é marginalmente significativo sobre $\triangle \mathrm{PIBI}$, ao nível de $10 \%$. Assim a taxa de variação do PIB da indústria da economia brasileira tem impactos relevantes sobre a sua própria taxa de variação, principalmente sobre a $\triangle$ PIBIT e também sobre a taxa de variação do PIB do comércio.

Enquanto é mais provável que o primeiro efeito (sobre $\Delta$ PIBIT) seja devido aos encadeamentos que a indústria tem com ela mesma e ao seu maior impacto sobre o nível de tecnologia, o segundo seria, provavelmente, devido aos seus efeitos na geração de renda e, desse modo, sobre o comércio no próximo período. De qualquer forma, para se ter uma melhor noção de como a taxa de crescimento do PIB industrial afeta os demais setores da economia brasileira, ou seja, quais são os canais, seria necessário um estudo mais aprofundado; o que foge do escopo do presente artigo.

Adicionalmente, a primeira defasagem de $\triangle$ PIBI tem um efeito positivo sobre a Formação Bruta de Capital Físico de Máquinas e Equipamentos. Assim, o efeito parece ser o 
inverso do modelo neoclássico proposto inicialmente por SOLOW (1956), ou seja, o efeito vai do crescimento da indústria para a acumulação de capital, pelo menos no caso de máquinas e equipamentos.

Outros estudos chegaram a conclusões semelhantes. Por exemplo, BLOMSTRÖM, LIPSEY e ZEJAM (1993), através de uma análise de mais de 100 países, encontraram evidências de que mudanças na taxa de crescimento econômico precedem as alterações ocorridas na taxa de formação de capital, considerando o período entre 1965 e 1985.

Uma explicação plausível para tal comportamento é a de que o crescimento econômico gera maiores oportunidades de investimento através da elevação de seus respectivos retornos. Assim, os resultados dão suporte à ideia de que as decisões de investimentos por parte dos empresários são endógenas e dependem das expectativas de retorno de seus projetos.

TABELA 3 - RESULTADOS DA ESTIMAÇÃO DO MODELO VAR: 1948 - 2007 (MODELO COMPLETO)

\begin{tabular}{lc|ccc|c|c|c|c}
\hline & $\Delta$ FBKF & $\Delta$ FBKFME & $\Delta$ PIB & $\Delta$ PIBA & $\Delta$ PIBI & $\Delta$ PIBIT & $\Delta$ PIBS & $\Delta$ PIBC \\
\hline$\Delta$ FBKF & 0.2168 & 0.3551 & -0.0664 & -0.2917 & -0.1008 & -0.1141 & 0.0426 & -0.0630 \\
& $(0.219)$ & $(0.348)$ & $(0.081)$ & $(0.2644)$ & $(0.180)$ & $(0.135)$ & $(0.124)$ & $(0.105)$ \\
$\Delta$ FBKFME & -0.1814 & -0.5315 & 0.0417 & 0.0454 & -0.0406 & 0.0183 & 0.1090 & 0.0299 \\
& $(0.127)$ & $(0.202)^{* * *}$ & $(0.047)$ & $(0.1534)$ & $(0.104)$ & $(0.078)$ & $(0.072)$ & $(0.061)$ \\
$\Delta$ PIB (L1) & -0.7115 & -5.4176 & 0.8983 & -0.1182 & 3.1806 & 1.0749 & 2.9011 & 0.0067 \\
& $(1.295)$ & $(2.054) * * *$ & $(0.481)^{*}$ & $(1.5608)$ & $(1.063)^{* * *}$ & $(0.798)$ & $(0.734)^{* * *}$ & $(0.623)$ \\
$\Delta$ PIBA & 0.0304 & 0.0291 & -0.0050 & -0.0975 & -0.0776 & 0.0280 & -0.0653 & 0.0136 \\
& $(0.132)$ & $(0.209)$ & $(0.049)$ & $(0.1592)$ & $(0.108)$ & $(0.081)$ & $(0.075)$ & $(0.063)$ \\
$\Delta$ PIBI (L1) & 0.1994 & 0.7666 & 0.1220 & 0.3419 & 0.2896 & 0.2245 & -0.1194 & 0.2067 \\
& $(0.224)$ & $(0.356)^{* *}$ & $(0.083)$ & $(0.2702)$ & $(0.184)$ & $(0.138)^{*}$ & $(0.127)$ & $(0.108)^{* *}$ \\
$\Delta$ PIBIT & 0.1845 & 1.3006 & -0.2464 & 0.6833 & -0.8733 & -0.5372 & -0.4266 & -0.0440 \\
& $(0.579)$ & $(0.918)$ & $(0.215)$ & $(0.6978)$ & $(0.475) *$ & $(0.357)$ & $(0.328)$ & $(0.278)$ \\
$\Delta$ PIBS (L1) & -0.0618 & -0.1627 & -0.1019 & 0.3878 & -0.1015 & -0.1412 & -0.3658 & -0.1157 \\
& $(0.269)$ & $(0.428)$ & $(0.100)$ & $(0.3250)$ & $(0.221)$ & $(0.166)$ & $(0.153) \mathrm{v}$ & $(0.130)$ \\
$\Delta$ PIBC (L1) & 0.9568 & 3.5036 & -0.0901 & -0.6009 & -1.1370 & 0.2300 & -1.5177 & 0.3578 \\
& $(0.843)$ & $(1.338)^{* * *}$ & $(0.313)$ & $(1.0164)$ & $(0.692)$ & $(0.520)$ & $(0.478)$ & $(0.406)$ \\
CONS & 0.0207 & 0.0656 & 0.0227 & 0.0034 & -0.0059 & 0.0153 & 0.0244 & 0.0294 \\
& $(0.023)$ & $(0.036 *$ & $(0.008) * * *$ & $(0.0275)$ & $(0.019)$ & $(0.014)$ & $(0.013)^{*}$ & $(0.011)^{* * *}$ \\
\hline
\end{tabular}

NOTAS: desvio-padrão entre parênteses. Um (1) asterisco indica que o coeficiente é significativo ao nível de $10 \%$, dois (2) indicam que é ao nível de $5 \%$, enquanto três (3) indicam significância ao nível de $1 \%$. Variáveis consideradas endógenas: $\triangle \mathrm{FBKF} ; \triangle \mathrm{FBKFME} ; \triangle \mathrm{PIB} ; \triangle \mathrm{PIBA} ; \triangle \mathrm{PIBI} ; \triangle \mathrm{PIBIT} ; \triangle \mathrm{PIBS} ; \Delta \mathrm{PIBC}$. De acordo com os critérios de seleção, foi utilizada apenas uma defasagem. Período 1948 - 2007 para dados anuais. Especificação com mais variáveis.

Outro resultado relevante apresentado na Tabela 3 é o efeito defasado do $\Delta$ PIB. Além de sua primeira defasagem (t-1) ter um impacto positivo sobre ela mesma em t, seu efeito sobre $\triangle \mathrm{PIBI}$ e $\triangle \mathrm{PIBS}$ também é positivo. Além disso, o efeito é considerável, visto que 
uma elevação da primeira defasagem de $\triangle \mathrm{PIB}$ em 1 p.p. eleva $\triangle \mathrm{PIBI}$ em 3,18 p.p e $\Delta$ PIBS em 2,9 p.p., sendo esses efeitos bem maiores do que o da primeira defasagem de $\Delta$ PIBI sobre $\Delta$ PIBIT (0,22 p.p.) e $\Delta$ PIBC (0,21 p.p), por exemplo.

Desse modo, a taxa de crescimento da economia como um todo tem um efeito de estimular os dois principais setores da economia brasileira, ou seja, o industrial e o de serviços. A inclusão do $\triangle \mathrm{PIB}$ se justifica assim, pois esse efeito não foi possível de ser visualizado nos resultados apresentados na Tabela 2. Controlando para as demais variáveis do modelo, a primeira defasagem de $\triangle \mathrm{PIB}$ ainda tem um impacto negativo sobre $\triangle \mathrm{FBKFME}$.

Finalmente, $\triangle \mathrm{PIBS}$ e $\triangle \mathrm{PIBC}$ têm impactos negativos sobre $\triangle \mathrm{PIBS}$. Sobre os outros setores da economia brasileira os coeficientes dessas duas variáveis não se mostraram significativos, o que indica que nenhum deles serve como motor de crescimento da economia brasileira no período 1948-2007. Apesar do impacto positivo de $\triangle \mathrm{PIBC}$ sobre a taxa de investimento de máquinas e equipamentos, os efeitos não se espalham por outros setores da economia e nem sobre o próprio comércio em períodos posteriores.

\section{CONCLUSÕES}

Os resultados apresentados mostram a importância do setor industrial sobre a taxa de crescimento da própria indústria, além de evidências de que ele é relevante na determinação da taxa de crescimento dos PIB da agropecuária e do comércio no período 1948-2007. Ele foi o único dos três setores (agropecuária, indústria e serviços) que se mostrou relevante em estabelecer encadeamentos positivos com ele mesmo e com outros setores da economia brasileira, ou seja, atuando como um motor do crescimento no período analisado.

Além disso, o artigo apresenta indícios de uma forte correlação entre o desempenho do setor industrial e o crescimento econômico brasileiro. Neste caso, o processo de mudança estrutural da economia brasileira a partir de meados dos anos 80 não representa um processo virtuoso no crescimento na medida em que o setor que vem perdendo participação é justamente aquele que tem maiores impactos positivos sobre a economia como um todo devido aos seus encadeamentos, ao maior processo de mudança tecnológica e aos efeitos sobre a demanda. 


\section{REFERÊNCIAS}

BLOMSTRÖM, M.; LIPSEY, R.; ZEJAN, M. Is fixed investment the key to economic growth? Centre for Economic Policy Research (CEPR) Discussion Papers, London, United Kingdom, n. 870, 1993. Disponível em: <http://www.cepr.org/pubs/dps/

DP870.asp>. Acesso em 02/03/2007.

ENDERS, W. Applied Econometric Time Series. Hoboken, New Jersey: John Wiley \& Sons, 2006.

FEIJÓ, C.A.; CARVALHO, P.G.M.; RODRIGUEZ, M.S. Concentração industrial e produtividade do trabalho na indústria de transformação nos anos 90: evidências empíricas. Economia: Revista da Anpec, n. 4, v. 1, p. 19-52, 2003

HIRSCHMAN, A. O. The strategy of economic development. New Haven: Yale University Press, 1958.

IPEADATA. Brasília. Disponível em: <http://www.ipeadata.gov.br>.

KALDOR, N. A model of economic growth. The Economic Journal, n. 67, v. 268, p. 591 624, 1957.

SILVA, M. V. B.; SILVEIRA NETO, R. M. Crescimento do emprego industrial no Brasil e geografia econômica: evidências para o período pós-real. Economia: Revista da Anpec, p. 117, 2007, no prelo.

SOLOW, R. M. A contribution to the theory of economic growth. The Quarterly Journal of Economics, n. 70, v. 1, p. 65-94, 1956.

SOLOW, R. M. Technical change and the aggregate production function. Review of Economics and Statistics, n. 39, v. 3, p. 312-320, 1957. 